\title{
Films of Halloysite Nanotubes Sandwiched between Two Layers of Biopolymer: From the Morphology to the Dielectric, Thermal, Transparency, and Wettability Properties
}

\author{
Giuseppe Cavallaro, D. Ines Donato, Giuseppe Lazzara,* and Stefana Milioto \\ Department of Chemistry “S. Cannizzaro”, University of Palermo, Viale delle Scienze, Parco D'Orleans II, 90128 Palermo, Italy \\ Supporting Information
}

ABSTRACT: This study focused on the preparation and characterization of films based on biopolymers (hydroxypropylcellulose and low methoxyl pectin) and halloysite nanotubes (HNTs). The morphology is strongly dependent on the polymer nature. In particular, we observed a sandwich-like structure composed of two hydroxypropylcellulose layers incorporating the HNTs and a homogeneous distribution of the nanotubes

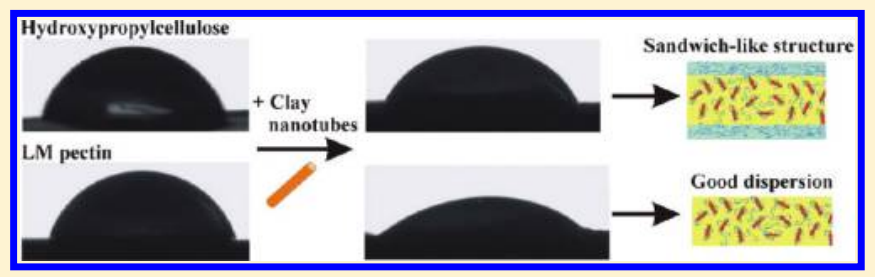
into the low methoxyl pectin matrix. The different mesoscopic properties were invoked to explain the dielectric, thermal, and wettability properties of the corresponding films. Nanocomposites obtained by combining materials from renewable resources and HNTs is a challenging task in view of designing green materials with tunable barrier and delivery properties for specific purposes.

\section{INTRODUCTION}

During the last few decades, materials based on nanoclays have attracted large interest because their properties and morphologies may be tuned. Within this field, halloysite nanotubes (HNTs) are newly emerging clays with unique features ${ }^{1}$ and appealing perspectives. ${ }^{2}$ Halloysite is a naturally occurring hydrated polymorph of kaolinite, and it possesses a peculiar hollow tubular shape, which results from the wrapping of clay layer. The size of HNTs is quite polydisperse, ranging between 0.1 and $2 \mu \mathrm{m}$, whereas the outer and inner diameters are ca. $30-50$ and 1-30 nm, respectively. ${ }^{1}$ The low cost and the environmentally friendly nature make the HNTs competitive with the carbon nanotubes in several applications. The HNT use in controlled release, nanotemplating, and sorption was recently reviewed. ${ }^{1}$ Loading the HNTs with anticorrosive agents for active coating protections was successfully done, and self-healing effect was achieved. $^{2-5}$ HNTs assembly by sequential adsorption with the polycations generated ordered multilayers. ${ }^{6}$ The selective recognition of contaminants was done by using molecular imprinted polymer based on magnetic HNTs. ${ }^{7}$ Because HNT is a green filler and a natural product, its use does not add any risk to the environment as other nanofillers in principle do. ${ }^{2}$ Polystyrene ${ }^{8}$ and polyamide 9 filled with well-dispersed HNTs exhibited a thermal and a mechanical reinforcement of the polymer matrix. Composites formed by clay tubes with nanoholes on the sidewalls were obtained by in situ mechanochemical polymerization of aniline in the presence of HNTs. ${ }^{10}$ In some cases, the functionalization of HNTs by grafting appropriate organic molecules was designed with the aim at obtaining a nanoclaybased filler with a high compatibility with the polymer matrix. ${ }^{11}$ Filling polymers from renewable resources with HNTs is a challenging task in view of designing new packaging materials.
A recent review ${ }^{12}$ reported that several biopolymeric materials may form films, but their use would be limited because of fast degradation and, in some cases, unsatisfactory mechanical properties. ${ }^{13}$ To our knowledge, very few insights into biopolymers filled with HNTs are available. ${ }^{14-17}$

Hydroxypropyl cellulose (HPC) obtained from the chemical modification of the cellulose may be an interesting biopolymer matrix of investigation. It was tested in several applications as a consolidant of waterlogged woods ${ }^{18}$ and drug delivery. ${ }^{19}$

Within this work, we prepared biofilms formed by HPC and HNTs, and we widely studied them by determining thermal stability, transparency, wettability, and dielectric properties. The morphology was helpful to explain the nanomaterial features. For a comparison purpose, some low methoxyl pectin/HNT composites were also investigated.

This work contributes to the knowledge on green nanocomposites, proving that materials with rather different mesoscopic structure can be designed by changing the biopolymer nature.

\section{EXPERIMENTAL SECTION}

Materials. Citrus pectin (with a low degree of methyl esterification, $\left.24 \%, M_{\mathrm{w}}=30-100 \mathrm{~kg} \mathrm{~mol}^{-1}\right)$ and $\operatorname{HPC}\left(M_{\mathrm{w}}=80 \mathrm{~kg}\right.$ $\mathrm{mol}^{-1}$ ) were from Aldrich. HNTs was a gift from Applied Minerals. All the materials were used without further purification. Water was from reverse osmosis (Elga model Option 3) with a specific resistivity $>1 \mathrm{M} \Omega \mathrm{cm}$.

$\begin{array}{ll}\text { Received: } & \text { July 29, 2011 } \\ \text { Revised: } & \text { September 7, } 2011 \\ \text { Published: } & \text { September 14, } 2011\end{array}$


Preparation of Nanocomposite Films. The aqueous polymer+filler mixtures were prepared as described elsewhere. ${ }^{14}$ In brief, citrus pectin (LM pectin) or HPC were solubilized in water under stirring at $70{ }^{\circ} \mathrm{C}$. The solutions were left to equilibrate at room temperature under stirring overnight. The final polymer concentration was evaluated by weight. Then, an appropriate amount of HNT was added to the polymer solution and kept under stirring overnight.

The well-dispersed aqueous mixture was poured in glass Petri dishes and heated at $80^{\circ} \mathrm{C}$ for $\sim 15 \mathrm{~h}$ to evaporate water until weight was constant. The films (with a thickness of ca. $0.06 \mathrm{~mm}$ ) were easily removed from the supports and stored in a desiccator at room temperature; their thickness was measured with a micrometer $\left( \pm 10^{-3} \mathrm{~mm}\right)$. The composition of nanofillers $\left(C_{\mathrm{f}}\right)$ expressed as weight percent (grams of filler/100 grams of nanocomposite) was systematically changed.

Methods. Dielectric Spectroscopy. A Hewlett-Packard impedance analyzer (HP 4294A) equipped with HP 16451B dielectric test fixtures was used at $25.0 \pm 0.1{ }^{\circ} \mathrm{C}$. The frequency was changed from $50 \mathrm{kHz}$ to $10 \mathrm{MHz}$. The dielectric constant $\left(\varepsilon_{\mathrm{r}}\right)$ and the dispersion factor $(\tan \delta)$ were measured, and the electrical conductivity $(\sigma)$ was calculated accordingly

$$
\sigma=\varepsilon_{\mathrm{r}} \varepsilon_{0} \omega \tan \delta
$$

where $\varepsilon_{0}$ and $\omega$ are the vacuum permittivity and the angular frequency of the alternating current, respectively.

Thermogravimetry. A Q5000 IR apparatus (TA Instruments) under a nitrogen flow of $25 \mathrm{~cm}^{3} \mathrm{~min}^{-1}$ for the sample and $10 \mathrm{~cm}^{3}$ $\min ^{-1}$ for the balance was used. The weight of each sample was ca. $10 \mathrm{mg}$. The measurements were carried out by heating the sample from room temperature to $900{ }^{\circ} \mathrm{C}$ at a rate of $10{ }^{\circ} \mathrm{C}$ $\mathrm{min}^{-1}$. From these experiments, the following parameters were determined: (1) the water content from the mass loss up to $150{ }^{\circ} \mathrm{C}$; $(2)$ the polymer decomposition temperature $\left(t_{\mathrm{d}}\right)$ taken at the maximum of the first-order derivative curves of mass loss to temperature (DTG curves); and (3) the residual weight at $900{ }^{\circ} \mathrm{C}$. The variation of the water content in the film with different HNT loadings is minute, and the residual weight clearly depends on $C_{\mathrm{f}}$. (Data are in the Supporting Information.)

Experiments were also performed to determine the kinetics of degradation of the polymer in the films. To this aim, two procedures were followed: (1) the nonisothermal degradation was studied by changing heating rates $\left(5,10,15\right.$, and $\left.20^{\circ} \mathrm{C} \mathrm{min}{ }^{-1}\right)$ and (2) the isothermal degradation was investigated by heating the sample to $310^{\circ} \mathrm{C}$ with a fast ramp $\left(500{ }^{\circ} \mathrm{C}\right.$ min $\left.^{-1}\right)$ and keeping the isothermal condition until a constant weight was registered.

Scanning Electron Microscopy. The morphology of nanocomposites was studied using a microscope ESEM FEI QUANTA 200F. Before each experiment, the surface of the sample was coated with gold in argon by means of an Edwards Sputter Coater S150A to avoid charging under electron beam. Minimal electron dose condition was set to avoid damage of the sample.

Films Transparency. The experiments were carried out at $25.0 \pm 0.1{ }^{\circ} \mathrm{C}$ by using an Analytic Jena Specord S 600 BU.

VIS-absorption spectra were recorded in the range from 400 to $800 \mathrm{~nm}$. The attenuation coefficient $(k)$ for each sample was computed as

$$
k=A /(2.3 \times D)
$$

where $A$ is the absorbance and $D$ is the thickness of the rectangular film measured with a micrometer $\left( \pm 10^{-3} \mathrm{~mm}\right)$. For all of the

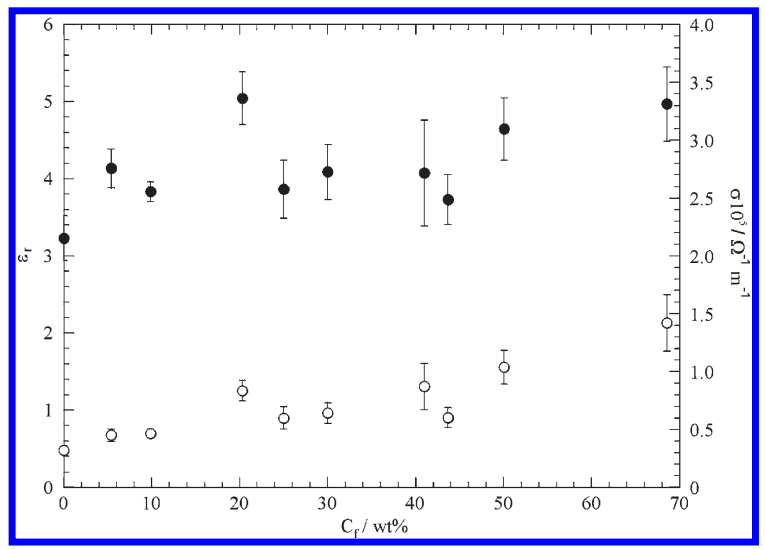

Figure 1. Dependence of the dielectric constant $(-)$ and the electrical conductivity $(\mathrm{O})$ of HPC/HNTs films on filler concentration.

investigated systems, the $k$ values are almost independent of $\lambda$ (examples are shown in the Supporting Information); therefore, the $k$ at $750 \mathrm{~nm}\left(k_{750}\right)$ will be discussed hereafter. The simplest way to analyze the $k_{750}$ data is based on the equation derived for spherical particles ${ }^{20}$

$$
k \propto \phi R
$$

where $R$ is the radius of the equivalent spherical particles scattering light and $\phi$ is their volume fraction. It has to be noted that the HNT particles do not have a spherical shape; therefore, $R$ assumes a more general meaning of an apparent radius of the spherical particle with the same scattering property of the nanotube.

Contact Angles. Contact angle measurements were performed by using an optical contact angle apparatus (OCA 20, Data Physics Instruments) equipped with a video measuring system having a high-resolution CCD camera and a high-performance digitizing adapter. SCA 20 software (Data Physics Instruments) was used for data acquisition. The films were fixed on top of a plane solid support and kept flat. The contact angle of water in air was measured by the sessile drop method. The water droplet volume was $6.0 \pm 0.5 \mu \mathrm{L}$. Temperature was set at $25.0 \pm 0.1^{\circ} \mathrm{C}$ for the support and the injecting syringe as well. Images were collected 25 times per second, starting from the deposition of the drop to $40 \mathrm{~s}$. From the data analysis, the contact angle, the volume, and the contact area of the drop were calculated. Five measurements at least were carried out on each film sample.

\section{RESULTS}

Dielectric Spectroscopy. This technique can provide information on the molecular dynamics of polymer systems monitoring the relaxation processes. Moreover, the dielectric properties of nanohybrids are of technological interest because they represent the capability to store/dissipate energy under the application of an electric field. Dielectric methods were extensively applied to cellulose and its derivatives. ${ }^{21-23}$ The dielectric spectroscopy studies for the HPC/HNTs mixtures showed that at a certain frequency HNTs addition hardly affect both $\varepsilon_{\mathrm{r}}$ and $\sigma$, which is consistent with the HNTs polar nature and the increased number of charge carriers in their presence (Figure 1).

It was established that HPC shows the relaxation processes related to the local chain motion via the glucosidic bond 


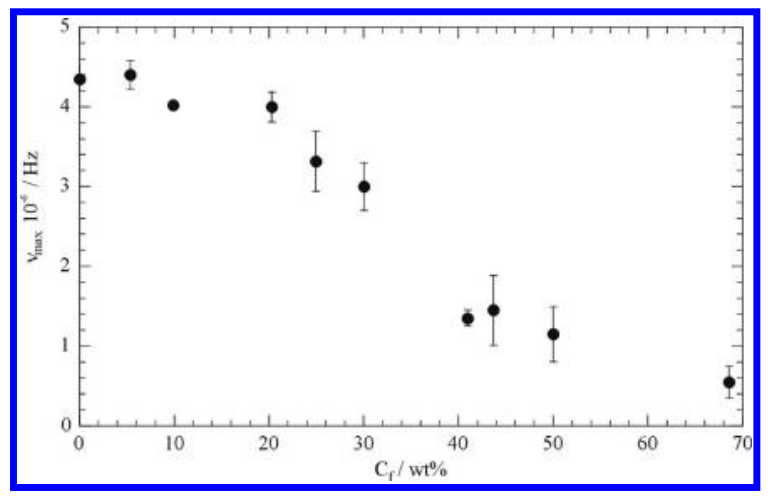

Figure 2. Relaxation frequency as a function of HNTs concentration for HPC/HNTs films.

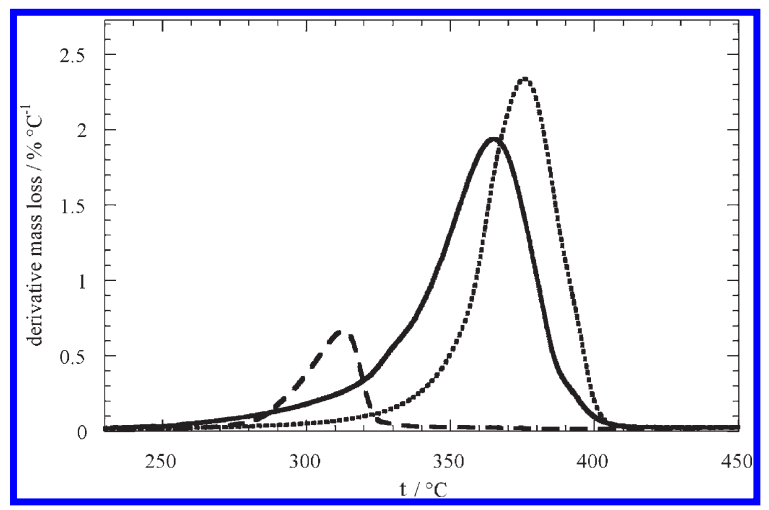

Figure 3. Thermal degradation rates as functions of temperature for HPC/HNTs films at $C_{\mathrm{f}}=0 \mathrm{wt} \%(-), 15 \mathrm{wt} \%(--)$, and $80 \mathrm{wt} \%(--)$.

( $\beta$-relaxation) and to the motion of the side groups attached to the glucosic unit of the polysaccharides ( $\gamma$-relaxation) both in the range from $10^{6}$ to $10^{7} \mathrm{~Hz}^{21}$ Very recently, dielectric spectroscopy evidenced free water relaxation at ca. $10^{10} \mathrm{~Hz}$ for wet HPC samples. ${ }^{22}$ In light of these insights, one may state that $\varepsilon_{\mathrm{r}}$ and $\tan \delta$ data as functions of the frequency $(v)$ for HPC in the absence and the presence of HNTs evidence the polymer relaxation (examples are in the Supporting Information). In particular, a maximum in the $\tan \delta$ versus $v$ trend is evident in the correspondence of the inflection point in the $\varepsilon_{\mathrm{r}}$ versus $v$ curve. The profile is strongly altered by the HNTs being that the peak is shifted toward lower frequencies and it becomes wider. A similar effect was observed for the dielectric response of $\mathrm{TiO}_{2}$-epoxy composite, and it was explained in terms of Maxwell-Wagner interfacial polarization. ${ }^{24}$ Interesting insights are provided by the relaxation frequency at the maximum of $\tan \delta\left(v_{\max }\right)$ versus $C_{\mathrm{f}}$ trend (Figure 2 ) because $v_{\max }$ slightly changes to $C_{\mathrm{f}} \approx 20 \mathrm{wt} \%$; thereafter, it sharply decreases upon the HNTs addition, reflecting a reduced local chain mobility in the presence of the nanotubes.

Thermal Degradation. Figure 3 reports DTG examples on the influence of HNTs on the thermal stability of HPC. Compared with the pristine HPC, the peak reflecting the polymer decomposition is shifted toward larger and lower temperatures for low and high $C_{\mathrm{f}}$ values, respectively. Such a peculiarity is clearly evidenced in the trend of $t_{\mathrm{d}}$ as a function of $C_{\mathrm{f}}$ (Figure 4). The presence of small amounts of nanofiller causes the HPC thermal stabilization (up to $10^{\circ} \mathrm{C}$ ), whereas a further

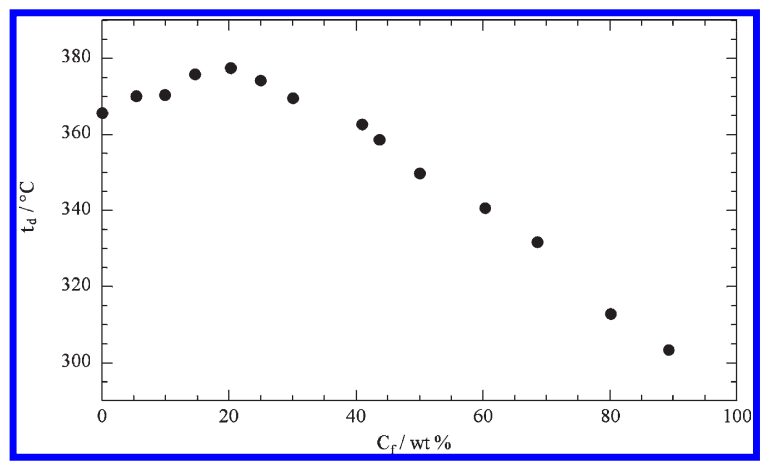

Figure 4. Degradation temperature as a function of the filler concentration for HPC/HNTs films.

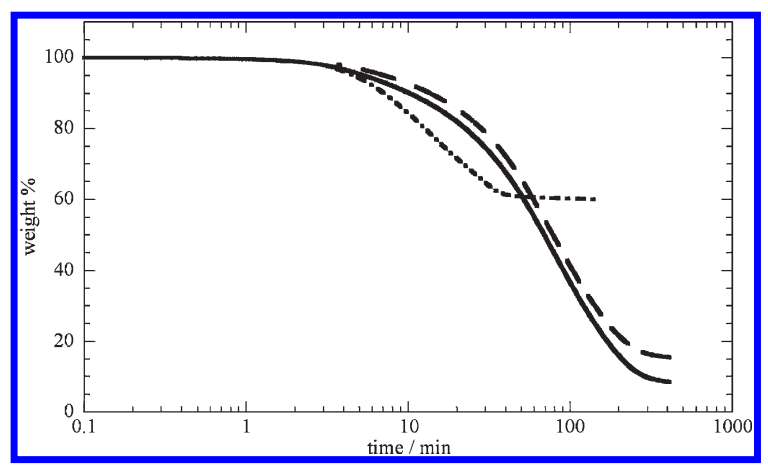

Figure 5. Isothermal degradation at $310^{\circ} \mathrm{C}$ for pristine $\mathrm{HPC}(--)$ and $\mathrm{HPC} / \mathrm{HNTs}$ films at $C_{\mathrm{f}}=10 \mathrm{wt} \%(--)$ and $60 \mathrm{wt} \%(-\bullet-)$.

addition of HNTs (above $C_{\mathrm{f}} \approx 20 \mathrm{wt} \%$ ) enhances the thermal degradation that occurs at temperature even $65{ }^{\circ} \mathrm{C}$ below $t_{\mathrm{d}}$ of pristine HPC. For LM pectin/HNTs mixtures, $t_{\mathrm{d}}$ sharply increases within the regime of very high HNT loading. ${ }^{14}$ The nanoparticles nature controls the thermal behavior of polymers. ${ }^{14,25-27}$ In the presence of HNTs the thermal stabilization is generally observed, ${ }^{14,25}$ and it is ascribed to the entrapment of the volatile products (obtained from the polymer thermal decomposition) into the HNTs lumen. Commonly, the effect on $t_{\mathrm{d}}$ of inorganic fillers in the polymeric matrices is discussed in terms of barrier effects toward both mass and heat transports; in particular, a composite material with well-dispersed nanoparticles typically shows a thermal enforcement. For a better understanding of the thermal degradation issue, we monitored the isothermal degradation at $310{ }^{\circ} \mathrm{C}$ for pristine HPC and nanocomposites at $C_{\mathrm{f}}=10$ and $60 \mathrm{wt} \%$. As Figure 5 shows, the mass is constant during an induction period; afterward, it decreases, approaching a plateau. Of course the residual mass is larger the higher the amount of inorganic nondegradable filler. Compared with pristine HPC, the thermal decomposition at $C_{\mathrm{f}}=10 \mathrm{wt} \%$ is slightly delayed, whereas it is strongly accelerated at $C_{\mathrm{f}}=60 \mathrm{wt} \%$; that confirms the $t_{\mathrm{d}}$ data.

Thermograms were also collected on the same samples at variable heating rates $(\beta)$ to determine the activation energy $\left(E_{\mathrm{a}}\right)$ of degradation. The Flynn-Wall-Ozawa approach as well as the Friedman's methods are nonisothermal and model-free, allowing us to calculate $E_{\mathrm{a}}$ as a function of the extent of conversion $(\alpha)$ without making any assumption on the reaction mechanism. For this reason, they are widely use $\mathrm{d}^{14,28-31}$ in kinetic studies of thermal decomposition of nanocomposites and macromolecules. 
In brief, the FWO method is based on the following integrated equation

$$
\ln \beta=\left[\frac{A E_{\alpha}}{R G(\alpha)}\right]-5.3305-1.0516 \frac{E_{\alpha}}{R T}
$$

where $G(\alpha)$ is a function of the extent of conversion, $A$ is the preexponential factor, $R$ is the gas constant, and $T$ is the absolute temperature. At a given $\alpha$ value, from the slope of the $\ln \beta$ versus $1 / T$ plot, one can easily calculate $E_{\mathrm{a}}$. As concerns the Friedman approach, the following equation was used

$$
\ln \left(\beta \frac{\mathrm{d} \alpha}{\mathrm{d} T}\right)=\ln [A f(\alpha)]-\frac{E_{\alpha}}{R T}
$$

where $\mathrm{d} \alpha / \mathrm{d} T$ is the first derivative of $\alpha$ with respect to temperature and $f(\alpha)$ is a function of the extent of conversion that depends on the degradation mechanism. In this case, the $E_{\mathrm{a}}$ values at each $\alpha$ value are obtained from the slopes of the $\ln (\beta \mathrm{d} \alpha / \mathrm{d} T)$ versus $1 / T$ plot.

It is worth noting that the results provided by the two methods are very similar, supporting the reliability of the data analysis. ( $E_{\mathrm{a}}$ data from the FWO method are in the Supporting Information.) For all of the investigated nanocomposites, $E_{\mathrm{a}}$ does not depend on $\alpha$ (Figure 6), but it does strongly depend on composition. At $C_{\mathrm{f}}=10 \mathrm{wt} \%, E_{\mathrm{a}}$ is slightly larger than the corresponding value of the pure HPC, whereas at $C_{\mathrm{f}}=60 \mathrm{wt} \%$, the energetic barrier to the degradation process drops to almost zero value.

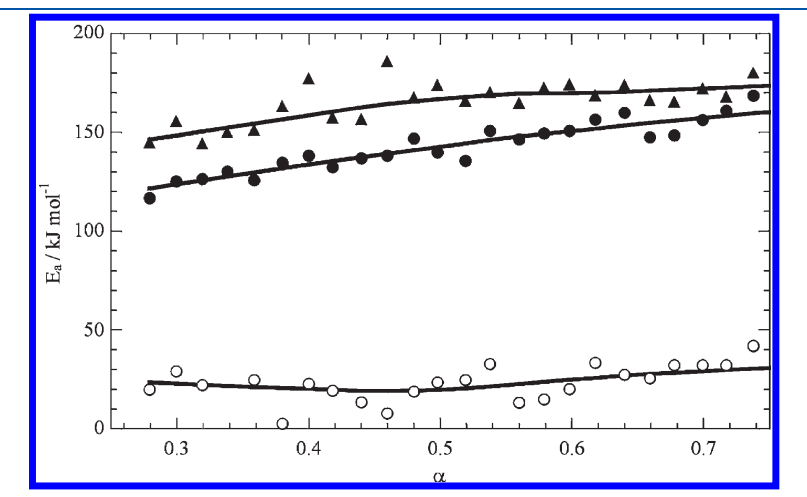

Figure 6. Activation energy as a function of the extent of conversion for the HPC/HNTs films at $C_{\mathrm{f}}=0 \mathrm{wt} \%(\bullet), 10 \mathrm{wt} \%(\boldsymbol{\Delta})$, and $60 \mathrm{wt} \%(\bigcirc)$ obtained from the Friedman method. Lines are guide for eyes.
Films Morphology. SEM experiments were carried out to image the morphology of the film both at the surfaces and at the transverse section. The morphology of the hybrid surfaces at both low and high HNTs content is very peculiar in the case of films based on HPC because the surface appears homogeneous and the presence of nanoparticles is rare (Figure 7). Being that in the film preparation, which is a solvent casting method followed by evaporation of the solvent, one surface of the film was exposed to air while the other surface was in contact with the glass Petri dish, in some cases, we performed measurements on both facesides of the film, and we did not evidence any difference on the general structure of the two sides (Figure 7). From these results, one may deduce that the sandwich-like structure is driven by interactions between the components of the films. This morphology is very different from that of LM pectin/HNTs films, which showed dispersed HNTs into the polymer matrix. ${ }^{14}$ Furthermore, such a difference is magnified when the micrographs obtained for the same HNTs composition and at the same sensitivity scale for the HPC/HNTs and LM pectin/HNTs systems are compared (Figure 8). On one side, the surface film of LM pectin evidences HNTs that are well-distinguishable and recognizable; on the other side, the surface film of HPC is in general very smooth, burying the nanotubes well. The peculiar morphology of the HPC/HNTs films induced us to perform measurements onto the edges of the films to check the distribution of the nanoclay along the lateral profile. Figure 9 shows that the nanotubes are well-compacted between two smooth thin layers of HPC in a sandwich-like structure. This amazing result is opposite to that reported ${ }^{14}$ for LM pectin/HNTs for which the nanoclay is equally distributed on the surface as well as along the edges.

In light of these findings, the interpretation of $k_{750}$ can be easily done. Figure 10 illustrates that for HPC/HNTs films the $k$ versus $\phi$ is a linear trend with a slope proportional to $R$ (eq 3 ), indicating that clustering between scattering object is negligible. For the pectins/HNT nanocomposites, the cluster formation is controlled by percolation, and it is enhanced for pectin with a higher degree of methyl-esterification. ${ }^{14}$

Wettability of HPC/HNTs Films. The surface properties of the HPC/HNTs and LM pectin/HNTs films were determined by measuring the contact angles $(\vartheta)$ of water as a function of time $(\tau)$ at variable HNTs composition. As a general result, $\vartheta$ decreases over time (examples are given in Supporting Information), whereas the drop volume and the contact area decreases and increases, respectively (Figure 11). Similar findings were obtained for surfaces

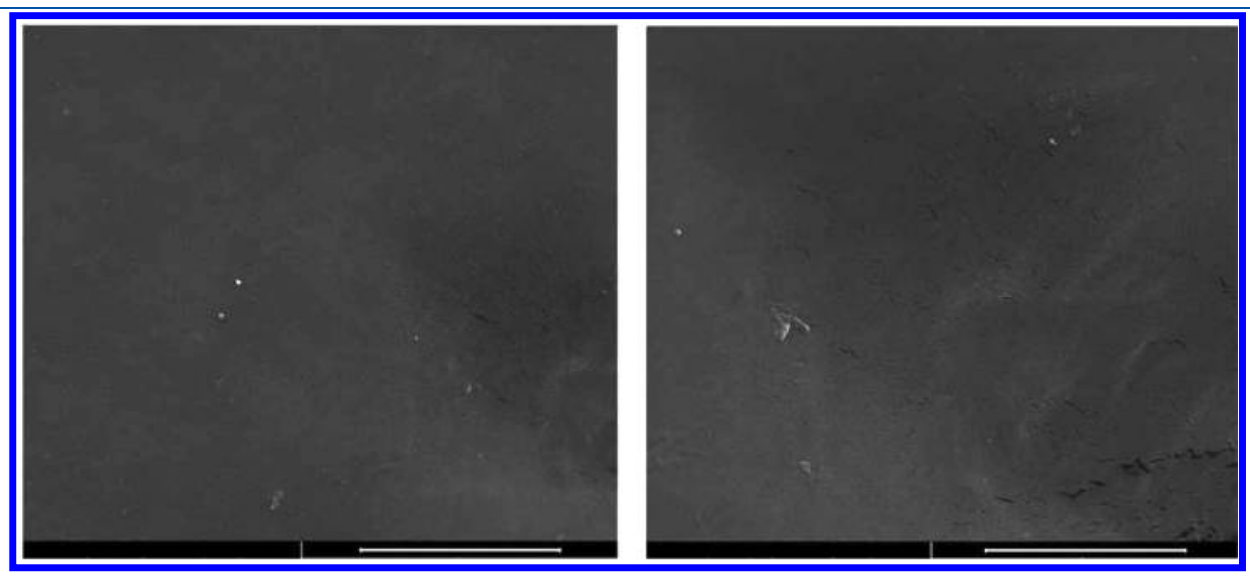

Figure 7. Scanning electron microscopy images of HPC/HNTs $\left(C_{\mathrm{f}}=50 \mathrm{wt} \%\right)$ for surface in contact with air (left-hand side) and glass Petri dish (righthand side). The bar is $10 \mu \mathrm{m}$. 


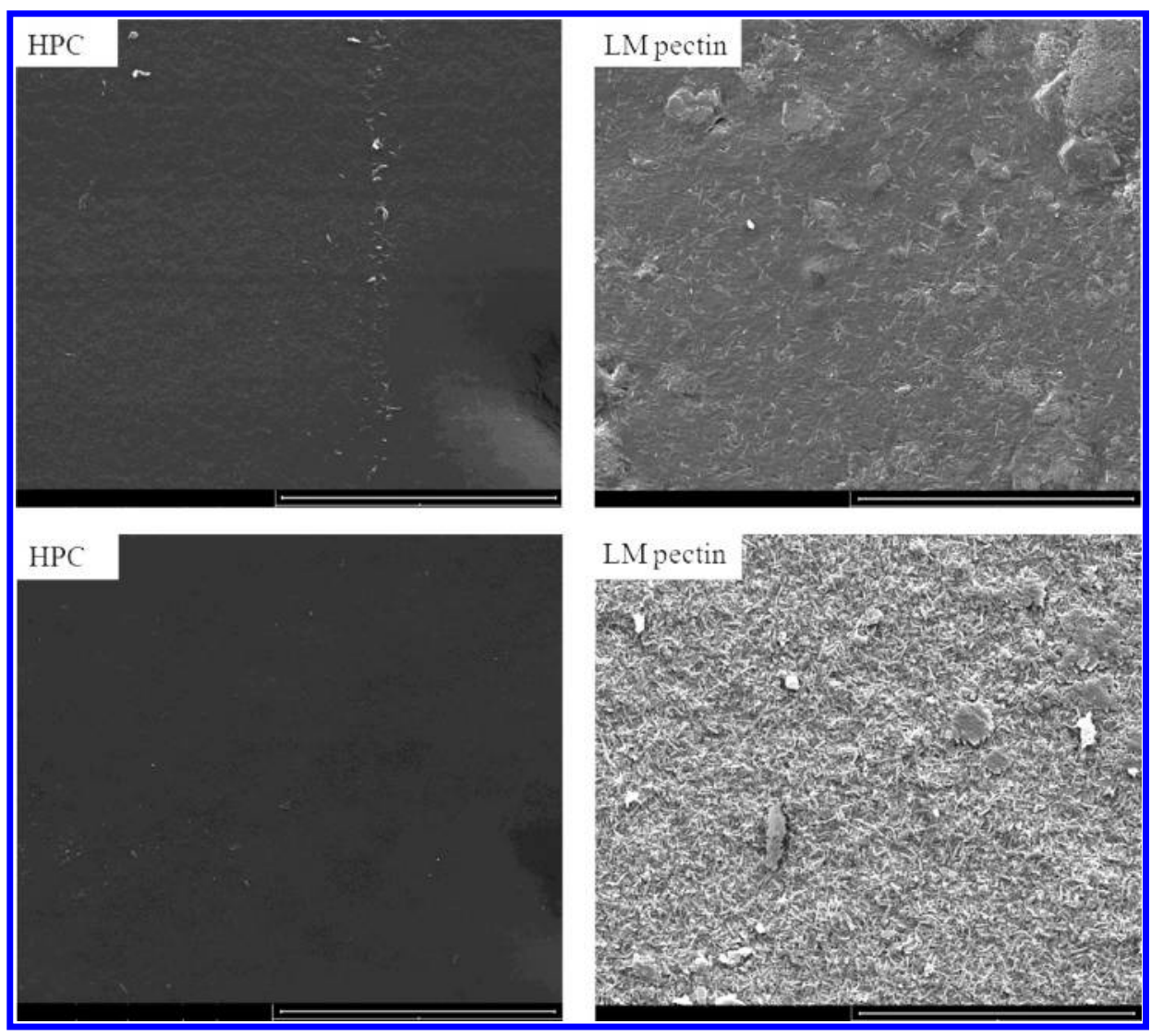

Figure 8. Scanning electron microscopy images of HPC/HNTs and LM pectin/HNTs films. The HNTs concentration is: top, $C_{\mathrm{f}}=10 \mathrm{wt} \%$; bottom, $C_{\mathrm{f}}=60 \mathrm{wt} \%$. The bar is $30 \mu \mathrm{m}$.

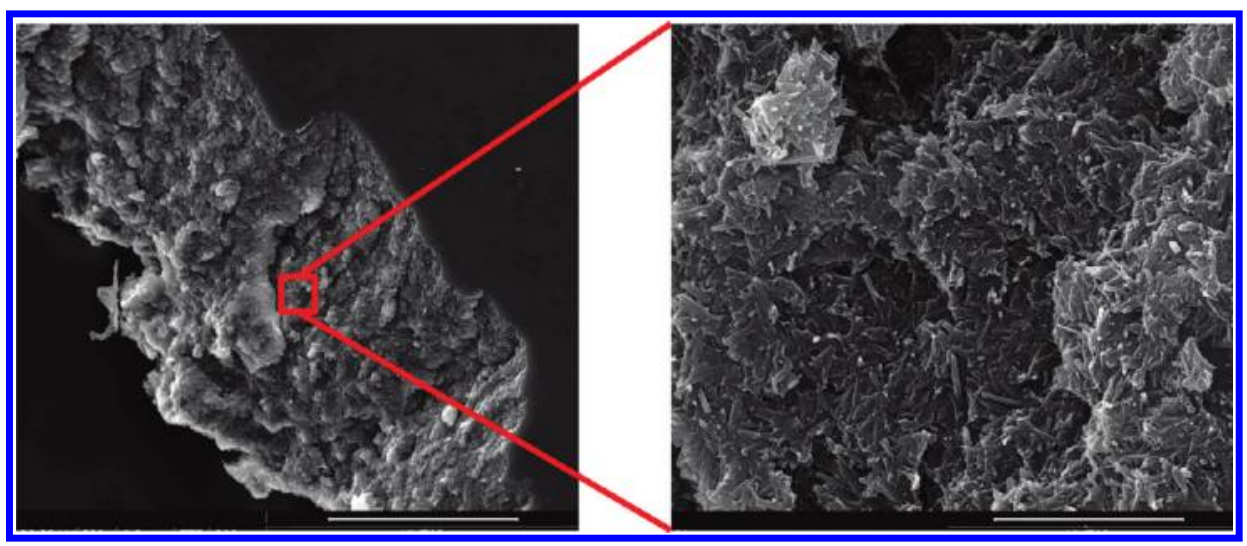

Figure 9. Scanning electron microscopy images of cross section of HPC/HNTs film at $C_{\mathrm{f}}=60 \mathrm{wt} \%$. The bar is $5 \mu \mathrm{m}$ at the right-hand side and $100 \mu \mathrm{m}$ at the left and side.

containing several biopolymers including pectin. ${ }^{32}$ By comparing the volume drop and the contact area data, we conclude that both spreading and water absorption occur during the experiment. ${ }^{32}$ A semiquantitative evaluation of the contributions from the spreading and the absorption can be done by fitting the $\vartheta$ versus $\tau$ curve as ${ }^{32}$

$$
\vartheta=\vartheta_{\mathrm{i}} \exp \left(k_{\vartheta} \tau^{\mathrm{n}}\right)
$$

where $\vartheta_{\mathrm{i}}$ is the contact angle at $\tau=0, k_{\vartheta}$ measures the process rate, and $n$ assumes fractional values ascribable to the occurrence of absorption and spreading. Equation 6 successfully fitted our data, providing $n$ values (Table 1 ) that reveal that both spreading and absorption take place; accordingly, $n=0$ and 1 for pure absorption and pure spreading, respectively. The negative values of $k_{\vartheta}$ reveal the decrease in contact angle.

The general trends of $\vartheta_{\mathrm{i}}$ versus $C_{\mathrm{f}}$ for both materials are rather intriguing (Figure 12). For LM pectin/HNT films, $\vartheta_{\mathrm{i}}$ is a decreasing function of $C_{\mathrm{f}}$, whereas for HPC/HNTs a peculiarity is exhibited by the $\vartheta_{\mathrm{i}}$ versus $C_{\mathrm{f}}$ trend, which remarks a welldefined minimum at $C_{\mathrm{f}}=15 \mathrm{wt} \%$. The large differences between the behavior of LM pectin- and HPC-based films can be also easily evidenced by the drop images collected just after deposition. (See the examples in Figure 13.) 


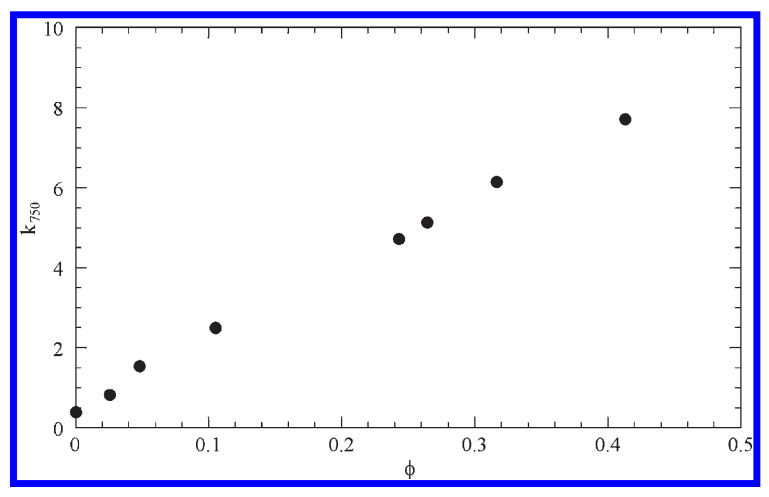

Figure 10. Dependence of the attenuation coefficient at $750 \mathrm{~nm}$ on the filler volume fraction for HPC mixed with HNTs.

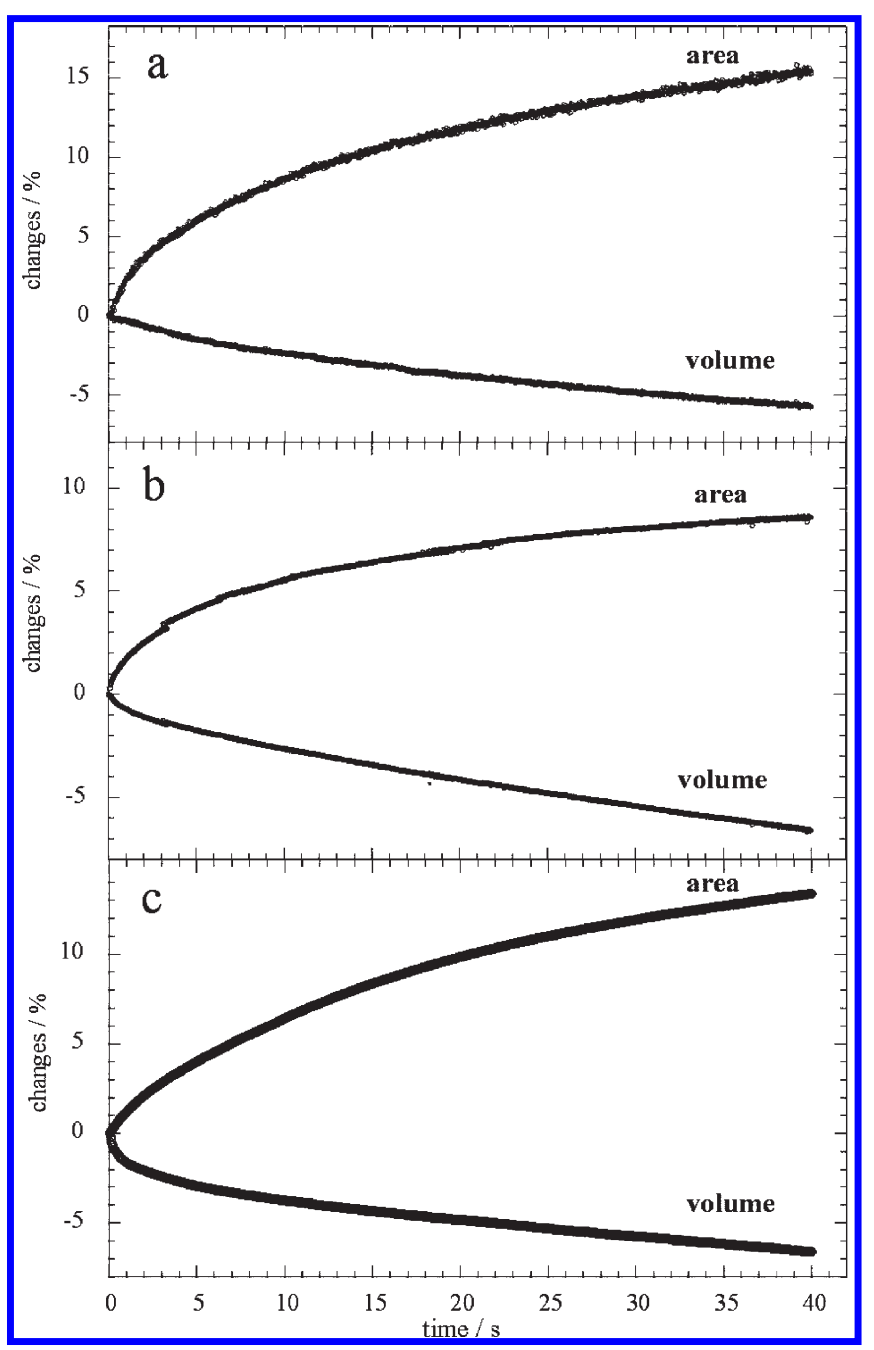

Figure 11. Water droplet volume and solid/liquid contact area evolution over time for HPC/HNTs at $C_{\mathrm{f}}=0$ wt \% (a), $30 \mathrm{wt} \%$ (b) and 60 wt $\%$ (c).

The changes in $\vartheta_{\mathrm{i}}$ can be related to the surface roughness or to the chemical hydrophilicity and therefore to the surface composition. Literature reports ${ }^{33}$ ascribed the enhancement in the surface hydrophilicity and surface free energy to the surface enrichment of the nanofillers (synthetic layered silicate as well as
Table 1. Fitting Parameters of Equation 6 Applied to the Contact Angle Data ${ }^{a}$

$\begin{array}{ccc}C_{\mathrm{f}} & k_{\vartheta} & n \\ & \text { LM pectin } & \\ 0 & -0.0103 & 0.425 \\ 9.88 & -0.0118 & 0.547 \\ 29.56 & -0.0152 & 0.431 \\ 60.27 & -0.0103 & 0.665 \\ & & \\ 0 & \text { HPC } & \\ 5.36 & -0.0296 & 0.482 \\ 9.85 & -0.0330 & 0.399 \\ 14.60 & -0.0076 & 0.555 \\ 20.29 & -0.0059 & 0.661 \\ 30.01 & -0.0279 & 0.475 \\ 50.02 & -0.0283 & 0.453 \\ 60.33 & -0.0204 & 0.380 \\ C_{6} & -0.0192 & 0.559\end{array}$

${ }^{a}$ Units are: $C_{\mathrm{f}}$, wt $\% ; k_{\vartheta}, \mathrm{s}^{-n}$. The errors are 1 and $0.3 \%$ on $k_{\vartheta}$ and $n$, respectively.

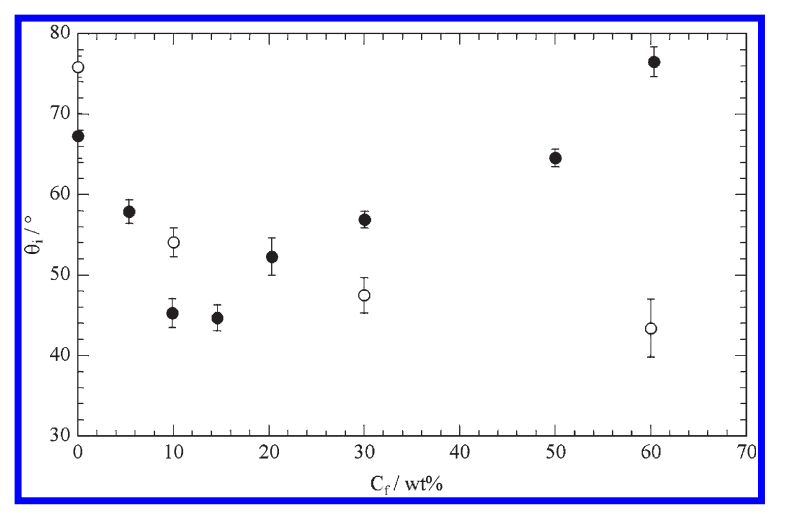

Figure 12. Dependence of the water contact angle at $\tau=0$ on the filler concentration for LM pectin/HNT (O) and HPC/HNT composites.

octaammonium polyhedral oligomeric silsesquioxane) dispersed in polyamide- 6 . The spherulite superstructures generating a very rough surface were indicated to be responsible for the superhydrophobic properties of the polypropylene/HNT nanocomposite surface; that agrees with the well-known Wenzel model describing the contact angle on a rough surface, for which the surface hydrophobicity is strictly correlated to the surface roughness. Furthermore, Marmur ${ }^{34}$ assessed that hydrophobic surfaces can be formed by hydrophilic substances if the roughness topography is multivalued.

\section{DISCUSSION}

The SEM images readily detected the difference on the surfaces and on the edges for the composite films of HPC and LM pectin. Moreover, TGA, dielectric, and wettability properties all indicated that they are affected by HNT loading. For the LM pectin/HNTs system, rather strong electrostatic interactions between the polymer and the nanofiller likely take place because of the presence of a certain number of carboxylic groups. This generates a sort of 


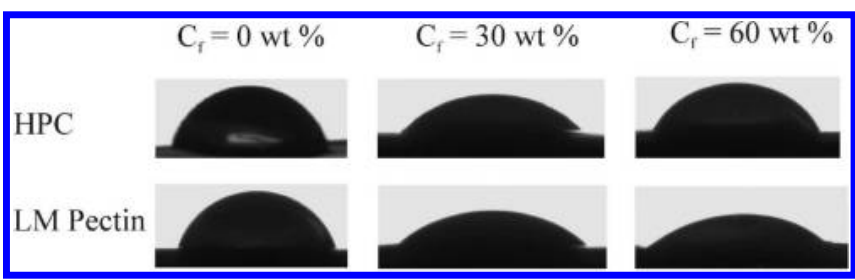

Figure 13. Images of water drops on films of biopolymer/HNTs at $\tau=0$.

polymer network where the nanotubes are well-dispersed; once the percolation regime for the HNT content is approached, the cluster formation takes place. ${ }^{14}$ In the HPC/HNTs films, electrostatic forces are absent; furthermore, the hydroxy-propyl groups cause the different morphology of the film. It likely occurs that HPC, due to the presence of the relatively hydrophobic hydroxypropyl groups, exhibits a smaller affinity toward HNTs; consequently, it generates a segregation between the HNTs and the polymer itself. In light of this picture, the other physicochemical properties can be understood.

From the dielectric spectroscopy, we evidenced that $v_{\max }$ exhibits two different behaviors occurring in the low and high concentration regimes (Figure 2): low amounts of HNTs do not alter the characteristic relaxation time, whereas a further HNT addition generates a reduced mobility of the polymer, which is related to the polymer confinement at the surface layers of the sandwich-like structure. We observe the enhancement of the thermal stability of HPC in the low HNTs region and surprisingly the enhancement of the thermal degradation in the high HNTs region. Furthermore, the activation energy for the degradation process is in line with the degradation temperature trends and shows that low HNT amounts increase the energetic barrier, whereas high HNT amounts essentially cancel it, speeding up the degradation process. These peculiarities cannot be correlated with a change in the morphology of HPC/HNTs films at different HNTs loadings because SEM images (Figures 8 and 9) did not reveal significant differences. For the HPC/HNTs films, transparency measurements indicated that the cluster formation is inhibited, and SEM images show that the nanotubes are well-dispersed along the plane directions of the film in the entire concentration range and are well-compacted between the HPC layers. To obtain a compact HNTs layer in the film, a certain concentration is needed; in fact, even if the nanotubes are not present at the film surface they may not be enough to generate a layer. From simple geometrical considerations one calculates that at $C_{\mathrm{f}}=10 \mathrm{wt} \%$ one may generate a HNTs layer of ca. $2 \mu \mathrm{m}$ (only two times the nanotube length) if the thickness of $60 \mu \mathrm{m}$ is considered for the film. On this basis, one can state that below that concentration the sandwich-like structure cannot exist because the HNT particle amount is low. Therefore, we argue that the HNTs layer is obtained above a certain $C_{\mathrm{f}}$ value and the sandwich-like structure makes available a reduced number of lumen able to incorporate volatile products generated by polymer. On the contrary, the homogeneous distribution of HNTs into the LM pectin matrix makes the nanofillers very good barriers, reinforcing the thermal stability of the films at high HNTs loadings. ${ }^{14}$

Finally, as concerns the wettability properties, the monotonic decrease of $\vartheta_{\mathrm{i}}$ with $C_{\mathrm{f}}$ observed for the pectin-based materials is in agreement with the enhancement of the surface hydrophilicity caused by the HNT enrichment at the interface as shown by SEM micrographs (Figure 8). The contact angle of $10^{\circ}$ for pure HNT was reported, ${ }^{2}$ confirming its hydrophilic nature.
For the HPC/HNT films, the peculiar increase of $\vartheta_{\mathrm{i}}$ starting at ca. $C_{\mathrm{f}}=15 \mathrm{wt} \%$ is not ascribable to the roughness of the surface ruled out by the SEM micrographs but rather to the rearrangement of the polymer structure that exposes the hydrophobic moieties to the interface as a consequence of the interaction with the inner HNTs layer. The HPC-based materials generally provide faster spreading/absorption than the corresponding film based on LM pectin. The sandwich-like structure is responsible for this discrepancy because the surface of the film is essentially a polymer layer.

\section{CONCLUSIONS}

Films composed of HNTs and polymers from renewable resources were prepared and characterized from the physicochemical viewpoint. The nature of the biopolymer exercises a key effect on the morphology. The hydroxypropylcellulose-based material forms a sandwich-like structure where the nanoclays are buried by two polymer layers. The LM pectin-based material is highlighted by a homogeneous HNTs dispersion on both the surface as well as on the transverse film section. The different mesoscopic structures of the film regulate the physicochemical properties.

Controlling the nanotube allocation within a biopolymericbased film can open the route to materials with interesting and tunable barrier and delivery properties useful for specific purposes.

\section{ASSOCIATED CONTENT}

S Supporting Information. Water content and residual matter for HPC/HNTs films from TGA data analysis. Dependence of the attenuation coefficient on the wavelength and roughness of the films. Dielectric constant and dispersion factor as functions of the frequency for HPC/HNTs films. Water contact angle as a function of time. Activation energy data from FWO method. This material is available free of charge via the Internet at http://pubs.acs.org.

\section{AUTHOR INFORMATION}

\section{Corresponding Author}

*E-mail: giuseppe.lazzara@unipa.it. Fax: +39 091590015. Phone: +390916459851.

\section{ACKNOWLEDGMENT}

The work was financially supported by the University of Palermo and COFIN 2008 (prot. 2008RH3FCW_002). Applied Minerals (USA) is acknowledged for kindly providing the Halloysite sample.

\section{REFERENCES}

(1) Du, M.; Guo, B.; Jia, D. Polvm. Int. 2010, 59, 574-582.

(2) Lvov, Y. M.; Shchukin, D. G.; Mohwald, H.; Price, R. R. $\underline{A C S}$ Nano 2008, 2, 814-820.

(3) Abdullayev, E.; Lvov, Y. L. Mater. Chem. 2010, 20, 6681-6687.

(4) Fix, D.; Andreeva, D. V.; Lvov, Y.; Shchukin, D. G.; Möhwald, H. Adv. Funct. Mater. 2009, 19, 1720-1727.

(5) Shchukin, D. G.; Lamaka, S. V.; Yasakau, K. A.; Zheludkevich, M. L.; Ferreira, M. G. S.; Mohwald, H. I. Phys. Chem. C 2008, $112,958-964$.

(6) Lvov, Y.; Price, R.; Gaber, G.; Ichinose, I. Colloids Surf. 2002, 198-200, 375-382. 
(7) Pan, J.; Yao, H.; Xu, L.; Ou, H.; Huo, P.; Li, X.; Yan, Y. L.Phys. Chem. C 2011, 115, 5440-5449.

(8) Lin, Y.; Ng, K. M.; Chan, C. M.; Sun, G.; Wu, J. I. Colloid Interface Sci. 2011, 358, 423-429.

(9) Lecouvet, B.; Gutierrez, J. G.; Sclavons, M.; Bailly, C. Polvm. Degrad. Stab. 2011, 96, 226-235.

(10) Zhou, C.; Du, X.; Liu, Z.; Mai, Y. W.; Ringer, S. L. Mater. Sci. 2011, 46, 446-450.

(11) Yuan, P.; Southon, P. D.; Liu, Z.; Green, M. E. R.; Hook, J. M.; Antill, S. J.; Kepert, C. J. L. Phvs. Chem. C 2008, 112, 15742-15751.

(12) Tharanathan, R. N. Trends Food Sci. Technol. 2003, 14, 71-78.

(13) Yu, L.; Dean, K.; Li, L. Prog. Polvm. Sci. 2006, 31, 576-602.

(14) Cavallaro, G.; Lazzara, G.; Milioto, S. Langmuir 2011, 27, $1158-1167$.

(15) Voon, H.; Bhat, R.; Easa, A.; Liong, M.; Karim, A. Food Bioprocess Technol. 2010, 1-9.

(16) Chang, P. R.; Xie, Y.; Wu, D.; Ma, X. Carbohvdr. Polvm. 2011, 84, 1426-1429.

(17) Xie, Y.; Chang, P. R.; Wang, S.; Yu, J.; Ma, X. Carbohvdr. Polvm. 2011, 83, 186-191.

(18) Giachi, G.; Capretti, C.; Macchioni, N.; Pizzo, B.; Donato, I. D. I. Cult. Heritage 2010, 11, 91-101.

(19) Guan, Y.; Zhang, Y.; Zhou, T.; Zhou, S. Soft Matter 2009, $5,842-849$.

(20) Pogodina, N.; Cerclé, C.; Avérous, L.; Thomann, R.; Bouquey, M.; Muller, R. Rheol. Acta 2008, 47, 543-553.

(21) Rachocki, A.; Markiewicz, E.; Tritt-Goc, J. Acta Phvs. Pol., A 2005, 108, 137-145.

(22) Sudo, S. I. Phvs. Chem. B 2011, 115, 2-6.

(23) Einfeldt, J.; Kwasniewski, A. Cellulose 2002, 9, 225-238.

(24) Fothergill, J. C. In Dielectric Polymer Nanocomposites; Nelson, J. K., Ed.; Springer: New York, 2010.

(25) Du, M.; Guo, B.; Jia, D. Eur. Polvm. I. 2006, 42, 1362-1369.

(26) De Lisi, R.; Lazzara, G.; Milioto, S.; Muratore, N. L. Therm. Anal. Calorim. 2007, 87, 61-67.

(27) Lazzara, G.; Milioto, S.; Gradzielski, M.; Prevost, S. L. Phvs. Chem. C 2009, 113, 12213-12219.

(28) Cavallaro, G.; Donato, I. D.; Lazzara, G.; Milioto, S. I. Therm. Anal. Calorim. 2011, 104, 451-457.

(29) Wang, S.; Tan, Z.; Li, Y.; Sun, L.; Li, Y. I. Therm. Anal. Calorim. 2008, 92, 483-487.

(30) Budrugeac, P.; Segal, E.; Perez-Maqueda, L. A.; Criado, J. M. Polvm. Degrad. Stab. 2004, 84, 311-320.

(31) Vyazovkin, S.; Dranca, I.; Fan, X.; Advincula, R. $\underline{\text { I.Phvs. Chem. B }}$ 2004, 108, 11672-11679.

(32) Farris, S.; Introzzi, L.; Biagioni, P.; Holz, T.; Schiraldi, A.; Piergiovanni, L. Langmuir 2011, 27, 7563-7574.

(33) Zhou, Q.; Pramoda, K. P.; Lee, J. M.; Wang, K.; Loo, L. S. I. Colloid Interface Sci. 2011, 355, 222-230.

(34) Marmur, A. Langmuir 2008, 24, 7573-7579. 